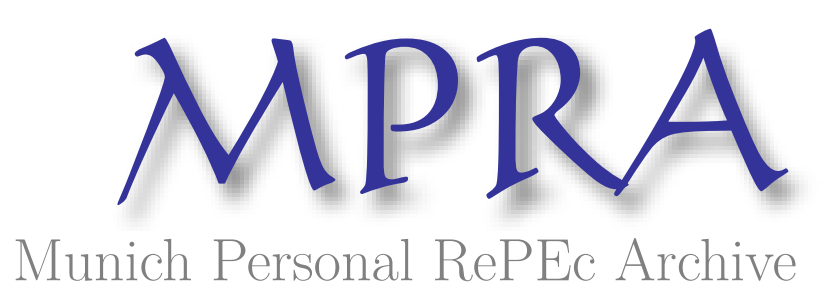

\title{
Modeling the price of crude oil and motor fuel: a five-year revision
}

Kitov, Ivan

Institute for Geospheres Dynamics

23 February 2015

Online at https://mpra.ub.uni-muenchen.de/62338/

MPRA Paper No. 62338, posted 25 Feb 2015 15:38 UTC 


\title{
MODELING THE PRICE OF CRUDE OIL AND MOTOR FUEL: A FIVE-YEAR REVISION
}

\author{
Ivan Kitov \\ Institute of Geospheres' Dynamics, Russian Academy of Sciences
}

\begin{abstract}
We present a five-year revision of an empirical study started in 2007. Seven years ago, we found two three distinct periods characterized by sustainable linear trends in the difference between the headline consumer price index (CPI) and the core CPI in the USA. Then we revealed similar behavior in the differences between the CPI and indices of various consumer expenditure categories. We estimated the duration of these trends which varies in a wide range from 5 years to more than 20 years. The transition periods to new trends span shorter intervals of 2 to 5 years. The transition is characterized by a higher level of volatility in the studied CPI differences. In April 2009, we introduced a simple quantitative model representing the evolution of motor fuel price (a subcategory of the consumer price index of transportation) relative to the core CPI as a linear function of time. Under our framework, all price deviations from this linear trend are transient and the price must return to the sustainable trend. The model predicted that oil price would fall to $\$ 30-\$ 60$ per barrel in 2016 , which is very close to the current price. The behavior of actual oil and motor fuel price since 2010 has shown that this prediction is accurate in both amplitude and trajectory shape - a good support for the credibility of our empirical mode. We conclude that the concept of price decomposition into a short-term (oscillating) and long-term (linear trend) components deserves a deeper theoretical consideration of the driving forces behind linear time trends and can be used as a workhorse for a wide spectrum of commodity investors. According to the model, the price of crude oil will be falling to the level of $\$ 30$ per barrel during the next 6 years and motor fuel will follow up the oil price. Moreover, the periodicity of the related normalized difference indicates that this low-price level may extend into the second half of the 2020s. The secular fall in energy prices may induce a lengthy period of very low inflation.
\end{abstract}

Key words: CPI, PPI, crude oil, motor fuel, price, prediction, USA JEL Classification: E31, E37 


\section{Introduction}

In 2009, we presented a model [1, 2] predicting the long-term evolution for various subcategories of consumer (CPI) and producer (PPI) price indices as well as for major commodities: crude oil, metals, grain, copper, etc. This model explicitly used one prominent feature of the difference between consumer (producer) prices of individual components and the overall consumer (producer) price index. In many cases, such differences are characterized by sustainable linear trends at a horizon of five to twenty years. For producer price indices, the reported trends are often slightly nonlinear but still robust. Here, we report that such trends were found and then confirmed for the price index of motor fuel (CPI component) [3] and crude oil (domestic production) [4]. This paper revisits our earlier consideration and predictions in order to demonstrate that the evolution of energy and oil price is well described by a series of linear trends with pivot points in 2000 and 2009. The next change in the price trend is not expected before 2018 and the era of low energy prices can be extended into the late 2020s.

As mentioned above, for major CPI and PPI subcategories these trends last from five to twenty years and then turn to trends with opposite slopes. We also found that the period of transition (often characterized by an elevated level of volatility) to new trends lasts three years at most $[1,2]$. However, there are several subcategories without slope changes as reported by the Bureau of Labor Statistics [5]. The best example of such one-leg trend since 1980 is the price index of medical care [1]. The index of communication has been linearly deviating from the headline CPI since 1998. In this study, we use seasonally adjusted CPIs and not seasonally adjusted PPIs.

In the short run, all actual prices oscillate around the long-term trends with varying amplitudes. The observed trends represent the lines of gravity centers for given prices and any large deviation from the trends must be compensated promptly. As a result, both short- and longterm predictions of commodity prices are feasible. In the long run, the prices follow up the trends. In the short-run, the next move in a given price depends on the current position relative to corresponding trend. When very far from the trend, the price is more likely to start it way back to the trend. When approaching the trend, the price may choose any direction for the further evolution, i.e. it should not inevitably go the other side of the trend. Using the revealed longterm trends and short-term deviations we predicted the evolution of prices for a number of consumer and producer price indices. 


\section{The model}

The model derived in [1,2] implies that the difference between the overall CPI (same for the PPI), $C P I(P P I)$, and a given individual price index $i C P I$ (iPPI), can be described by a linear time function over time intervals of several years:

$$
C P I(t)-i C P I(t)=A+B t
$$

where $A$ and $B$ are the regression coefficients, and $t$ is the elapsed time. Therefore, the "distance" between the CPI and the studied index is a linear function of time, with a positive or negative slope $B$. Free term $A$ compensates the difference related to the start levels of the involved CPIs for a given year. For example, the index of communication was started from the level of 100 in December 1997 when the overall CPI was already at the level of 161.8 (base period 1982-84 $=100)$.

Figure 1 displays two time series for the differences between price indices related to the scope of this paper and highlights the relevant periods with sustainable linear trends. In the left panel, the evolution of the index of motor fuel relative to the core CPI is shown. In the original paper [1], data were available only for the period before 2009 and the original empirical approach was developed in 2007 when the difference was far from its minimum in 2008 when the highest oil price was observed. In [1], our general expectation for the future was formulated in two statements. Firstly, the difference had to reach its lowermost value in 2008 and then the linear trend observed after 2002 had to turn to a new trend with an opposite slope. We considered the possibility of a "mirror reflection" of the 2002-2008 trend, i.e. that the new slope would have the same absolute value. The expected new trend is also shown by dashed line in the left panel of Figure 1. Secondly, a period of higher volatility in energy price was expected. Both predictions were accurate in quantitative terms - the trend started to turn in 2009 and the period of extremely high volatility lasted from 2008 to 2011. In 2010 [4], we reported that the new trend started to emerge since the beginning of 2010 and supposed that its duration would be at least seven years. Therefore, the difference should increase from -50 in the beginning of 2009 to +75 in 2016 since the index of motor fuel would be falling at a rate of 21 units per year (the new trend) relative to the core CPI. This was the long-term prediction of the motor fuel index. In reality, the trend reached 2011 and the level of -110 and then the current trend started. We drew the new trend in Figure 1 following up the instructions given in 2009. The new prediction is based on the 
observations between 2010 and 2015 and extends the trend into 2020, when the difference will reach 80 .

Currently, one can distinguish in Figure 1 three distinct periods of linear dependence on time: from 1980 to 1999, from 2001 to 2008, and from 2011 onwards. Linear regression of the CPIs' difference for two periods of sustainable linear trends gives high $\mathrm{R}^{2} \sim 0.9$. The current trend in the difference started in 2011 and has been evolving with large fluctuations. We drew the new trend as a "mirror reflection" of the trend observed from 2002 to 2008. After 2011, there was a period of low growth rate and the difference stayed below the predicted trend. Lately, the actual time series returned to the expected trend and likely will stay above the trend for several months. There are two finished transition periods: between 1999 and 2002 and from 2008 to 2011. Notice that there was only one finished transition period in 2009 when [1] was published. Between 1999 and 2002, the original trend with a positive slope $(B=+4.2)$ changed to a negative one $(B=-$ 21.1).
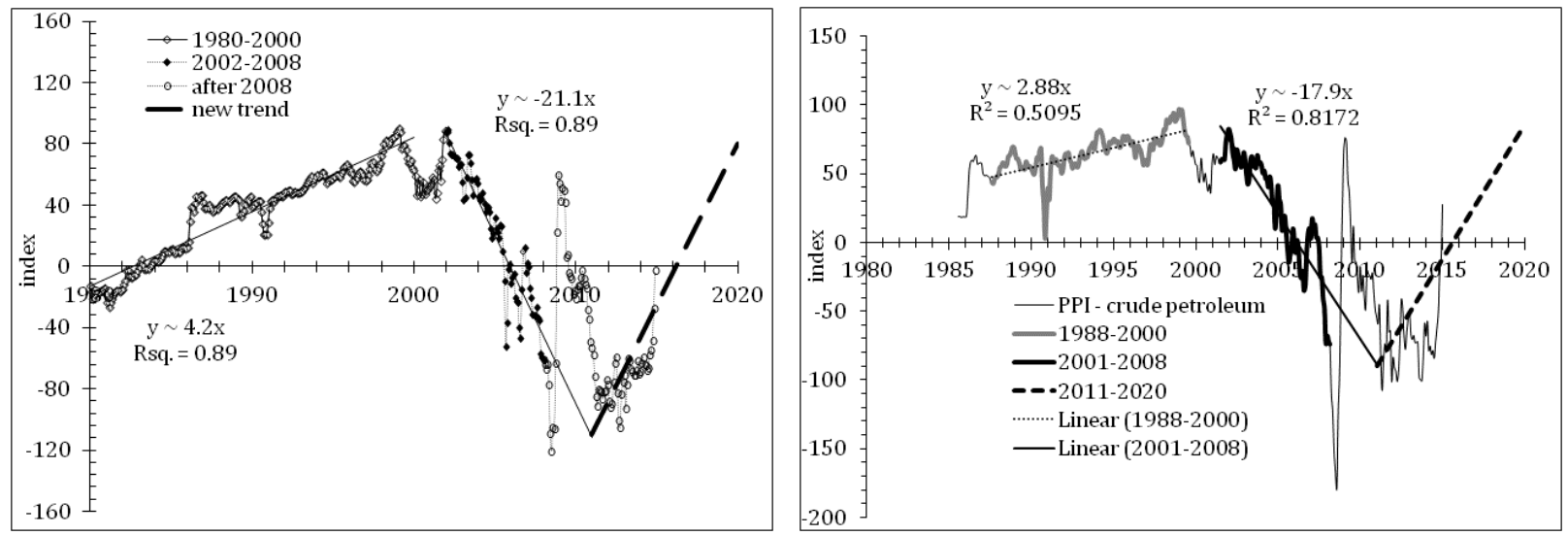

Figure 1. Illustration of linear trends. Left panel: the difference between the headline CPI and the index of motor fuel between 1980 and 2015. Right panel: The difference between the overall PPI and the (producer price) index of crude petroleum (domestic production).

In the right panel of Figure 1, the difference between the PPI and the index of crude petroleum (domestic production) is shown between 1985 and 2015. There are two distinct periods of linear dependence on time: from 1988 to 1999 and from 2001 to 2008, and the trend expected after 2011 is not as clear as the trend in the CPI difference in the left panel. The regression coefficients in the two past periods are different from those for the index of motor fuel: +2.9 and -17.9 , respectively. In other words, the motor fuel index slightly over-reacts the change in oil price. There was two transition periods between 1999 and 2001 and from 2008 to 
2011. The original negative trend between 2001 and 2008 finally turned to the current positive trend in 2011. Even simple visual inspection of the transition periods reveals several differences in timing and amplitude between motor fuel and crude oil. During the first transition period, the former started to fall three months later and came to the level of 1999 in the end of 2001.

From Figure 1, one can conclude that the presence of linear trends is a basic feature of the CPI and PPI. Another fundamental characteristic of the differences consists in the fact that all deviations from the trends were only short-term ones. This implies that any current or future deviations from the new trends in Figure 1, which have been evolving since 2011, must be compensated before the new trend will emerge. According to the trend in Figure 1, the price of crude oil will be falling to the level of $\$ 20$ to $\$ 30$ per barrel during the next 6 years and motor fuel will follow up the oil price.

\section{Cycles in energy price}

We used only the absolute difference between consumer and producer price indices so far. It is instructive to analyze the difference in relative terms. Instead of motor fuel and crude oil we selected the consumer price index of energy representing a broader spectrum of energy related commodities and categories. We have normalized the difference of the energy CPI and core CPI to the core CPI. Black curve in the left panel of Figure 2 illustrates the new pattern. In contrast to Figure 1, the amplitudes and periods of long term fluctuations are similar and the overall evolution seems to be repeatable, i.e. cyclic. Red curve in Figure 2 exercises the assumption of repeatability. We have shifted the original curve by 27 years ahead and obtained a striking similarity in the amplitude and timing of the energy price falls and rises. Right panel of Figure 2 shows a detailed picture, which could be helpful in the estimation of the lowermost value of the energy index relative to the core CPI. The current difference presented by black curve is expected to reach red line in during the current phase of energy price fall. Two years ago we forecasted a fast downfall in energy price and it is here today [6]. When the black line touch the red one - the energy index returns to the long term sustainable trend stretching into the 2020s. The era of low energy prices has come and the trend might not change to the opposite before, say, 2025. 

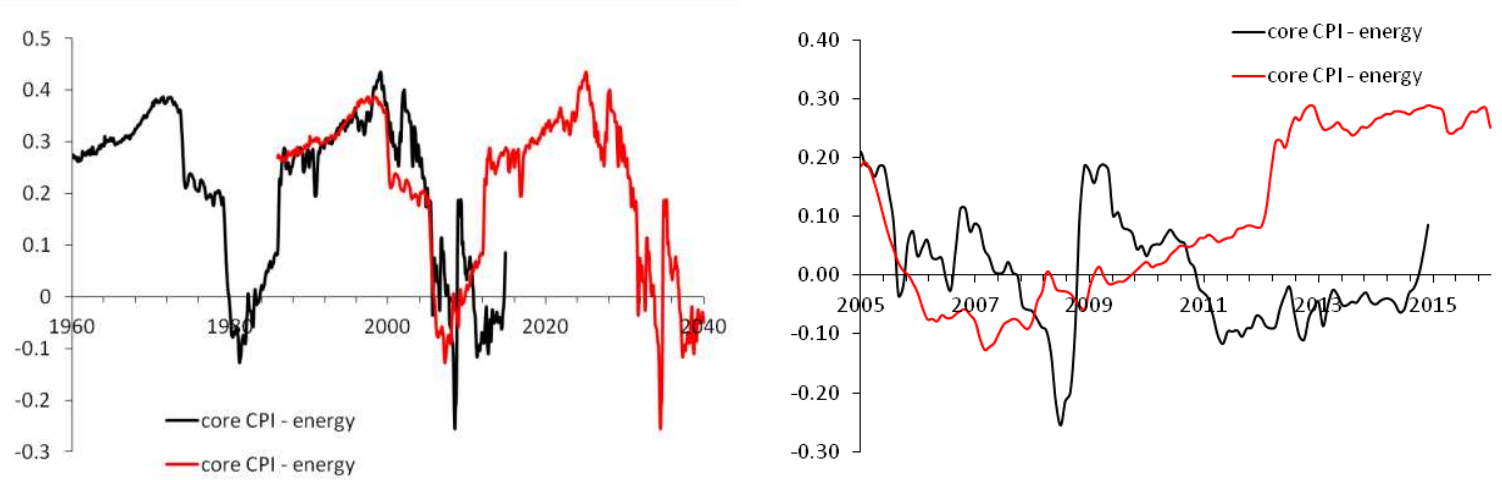

Figure 2. Left panel: Black line - the difference between the core and energy CPIs normalized to the core CPI. Red curve is representing the original (black) curve shifted 27 years ahead. Right pane: The energy fall is not over.

\section{Discussion}

All in all, our prediction of the price index of motor fuel was based on a sound assumption and proved its overall accuracy. The forces behind the observed long- and short-term behavior are not accessible yet but very powerful. We dare say they are fundamental and affect the economy to its deepest roots. These forces retain equilibrium among all economic agents and originate the sustainable trends in the differences between consumer (producer) price indices. At some point, the forces meet their limits and should be re-balanced in order not to harm the economy. As a result, the sustainable trends in the CPI and PPI turn. The results of seven-year observations and modeling under our framework deserve a deeper theoretical consideration of the driving forces behind linear time trends. Investors can use the model as a workhorse for a wide spectrum of commodities.

Meanwhile, it is instructive to revise our long-term prediction of oil price shown in Figure 1. After a major adjustment observed in 2014, the index of motor fuel and crude petroleum in Figure 1 show that our previous prediction (from 2009 and 2010) of oil price $\$ 30$ to $\$ 60$ per barrel in 2016 does not seem unreasonable. When following the new trend, oil price may reach $\$ 20$ to $\$ 30$ in 2020 . The index of motor fuel will follow up the trend with a delay and larger amplitude. Short-term fluctuations can not be predicted at a horizon of several years. However, the larger is a given deviation from the trend the larger is the returning force. 
Correspondingly, the energy consumer price will be falling another five to six years before it will reach its bottom. Considering the possibility of cyclic evolution, the era of low energy price may extend into the 2020s. The low energy price will force the overall fall in various consumer prices casing a longer period of extremely low price inflation and even deflation. The change in energy price might be also characterized by an elevated level of volatility.

\section{References}

1. Kitov, I., Kitov, O. (2008). Long-Term Linear Trends In Consumer Price Indices, Journal of Applied Economic Sciences, Spiru Haret University, Faculty of Financial Management and Accounting Craiova, vol. III(2(4)_Summ), pp. 101-112.

2. Kitov, I., Kitov, O. (2009). Sustainable trends in producer price indices, Journal of Applied Research in Finance, vol. I(1(1)_S Summ), pp. 43-51.

3. Kitov, I., Kitov, O. (2009). A fair price for motor fuel in the United States, MPRA Paper 15039, University Library of Munich, Germany.

4. Kitov, I., Kitov, O. (2010). Crude Oil and Motor Fuel: Fair Price Revisited, MPRA Paper 21869, University Library of Munich, Germany.

5. Bureau of Labor Statistics. (2010). Databases, Tables \& Calculators by Subject, retrieved 30.03.2010 from http://www.bls.gov/data/

6. Kitov, I., Kitov, O. (2010). Sustainable trends and periodicity in consumer price indices indicate that the era of low energy prices is approaching, MPRA Paper 43392 University Library of Munich, Germany. 